\title{
Impact of the Nationwide Intravenous Selenium Product Shortage on the Development of Selenium Deficiency in Infants Dependent on Long-Term Parenteral Nutrition \\ 全国性静脉注射硒产品短缺对依赖长期肠外营养的婴儿硒缺乏的影响
}

Connie H. Chen, PharmD, MEng1

Mary Beth Harris, MPH, RD, CSP $\underline{2}$

M. Luisa Partipilo, PharmD, BCNSP $\underline{3}$

Kathleen B. Welch, MS, MPH$\underline{4}$

Daniel H. Teitelbaum, MD $\underline{5}$

Allison B. Blackmer, PharmD, BCPS $\underline{6}, \underline{7 \Uparrow}$

${ }^{1}$ College of Pharmacy, University of Michigan, Ann Arbor, Michigan, USA

${ }^{2}$ Children's Intestinal Rehabilitation Program, University of Michigan C.S. Mott

Children's Hospital, Patient Food and Nutrition Services, Ann Arbor, Michigan, USA

${ }^{3}$ Intestinal Rehabilitation/Homemed, College of Pharmacy, The University of Michigan Health Systems, C.S. Mott Children's and Women's Hospital, Ann

Arbor, Michigan, USA

Allison B. Blackmer, PharmD, BCPS, Assistant Professor of Pharmacy, University of Colorado Anschutz Medical Campus, Skaggs School of Pharmacy and Pharmaceutical Sciences, Department of Clinical Pharmacy, Mail Stop C238, 12850 E. Montview Blvd. Aurora, CO 80045, USA. Email: allison.blackmer@ucdenver.edu.

\section{摘要}

背景：对于依赖肠外营养（PN）的患儿, 必须在静脉内补充硒。全国范围内的静脉硒短缺 始于 2011 年四月。目的是通过检查硒的提供, 生化性硒缺乏的发生, 和短缺相关的费用, 来评估硒产品短缺对 PN 依赖贞儿的影响。材料和方法: 这一单一中心, 回顾性研究包 括 : 年龄 $\leqslant 1$ 岁, 体重 $\leqslant 30 \mathrm{~kg}$ 的 依赖 PN 的患儿, 已接受 $\mathrm{PN} \geqslant 1$ 月, 并且已有 $\geqslant 1$ 次血 清硒测量。主要结局为生化性硒缺乏的发生率。次级结局包括生化缺陷的严重程度, 临床 表现, 成本和血清硒水平和硒剂量之间的关系。结果: 与非短缺时期 $(3.8 \pm 1 \mu \mathrm{g} / \mathrm{kg} / \mathrm{d}$; 范围, $2.4-6 \mu \mathrm{g} / \mathrm{kg} / \mathrm{d} ; \quad P<.001)$ 比较, 短缺期间平均硒剂量减少 2 倍 $(2.1 \pm 1.2$ $\mu \mathrm{g} / \mathrm{kg} / \mathrm{d}$; 范围， $0.2-4.6 \mu \mathrm{g} / \mathrm{kg} / \mathrm{d}$ ）。血清硒浓度与硒剂量之间呈现线性关系

$\left(r^{2}=0.42\right)$; 在大多数情况下, 剂量 $6 \mu \mathrm{g} / \mathrm{kg} / \mathrm{d}$ 的预期导致正常血清水平。两组病人发 生生化性硒缺乏的比例相似: 短缺时期, 59.1\%; 非短缺时期, $66.7 \% ; P=.13$ 。生化 性硒缺陷的严重程度两组相似。观察到在短期期间增量成本显著增加。结论：这是第一个 研究静脉注射硒短缺对依赖肠外营养的贞儿的影响。两组均表现出同样的生物硒缺乏的高 发病率, 提示经验性高剂量可能使这些患儿收益。然而, 持续的短缺限制了补充。 